\title{
Multiscaling in Hall-Magnetohydrodynamic Turbulence: Insights from a Shell Model
}

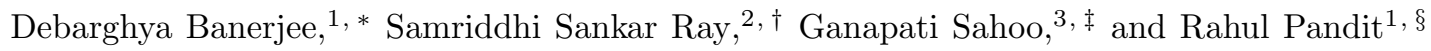 \\ ${ }^{1}$ Centre for Condensed Matter Theory, Department of Physics, \\ Indian Institute of Science, Bangalore 560012, India \\ ${ }^{2}$ International Centre for Theoretical Sciences, Tata Institute of Fundamental Research, Bangalore 560012, India \\ ${ }^{3}$ Max Planck Institute for Dynamics and Self-Organization, Am Fassberg 17, 37077 Göttingen, Germany
}

(Dated: August 17, 2021)

\begin{abstract}
We show that a shell-model version of the three-dimensional Hall-magnetohydrodynamic (3D HallMHD) equations provides a natural theoretical model for investigating the multiscaling behaviors of velocity and magnetic structure functions. We carry out extensive numerical studies of this shell model, obtain the scaling exponents for its structure functions, in both the low- $k$ and high- $k$ powerlaw ranges of 3D Hall-MHD, and find that the extended-self-similarity (ESS) procedure is helpful in extracting the multiscaling nature of structure functions in the high- $k$ regime, which otherwise appears to display simple scaling. Our results shed light on intriguing solar-wind measurements.
\end{abstract}

PACS numbers: 52.35.Ra, 95.30.Qd

Keywords: Hall-MHD, turbulence, multiscaling

Turbulent plasmas abound in accretion disks, galaxies, stars, the solar wind, and laboratory experiments [1, 2]; thus, the characterization of the statistical properties [1, [3, 4] of turbulence in such plasmas is a problem of central importance in astrophysics, plasma physics, fluid dynamics, and nonequilibrium statistical mechanics. Such a characterization begins with the energy spectra: e.g., in homogeneous and isotropic fluid turbulence the energy spectrum $E(k)$, which gives the distribution of energy over different wave numbers $k$, assumes the scaling form $E(k) \sim k^{-\alpha}$ if the Reynolds numbers $R e$ is large and $k$ is in the inertial range $L^{-1} \ll k \ll k_{d}$, where $L$ is the energy-injection length scale and $k_{d} \equiv 2 \pi / \eta_{d}$, with $\eta_{d}$ the length scale at which viscous dissipation becomes significant; the phenomenological theory of Kolmogorov (K41) yields [5, 6] $\alpha=5 / 3$. Turbulent plasmas show similar scaling forms for the kinetic and magnetic-energy spectra $E^{u}(k)$ and $E^{b}(k)$, if the turbulence is statistically homogeneous and isotropic, and both $R e$ and the magnetic Reynolds numbers $R e_{M}$ are large; their ratio $\operatorname{Pr}_{M}=R e_{M} / R e$, the magnetic Prandtl number, governs the relative sizes of the fluid and magnetic dissipation length scales $\eta_{d}^{u}$ and $\eta_{d}^{b}$; the inertial-range scaling properties of $E^{u}(k)$ and $E^{b}(k)$ have been studied theoretically and numerically by using the equations of magnetohydrodynamics (MHD) 1, 3, 4. Energy-spectra measurements in the solar wind [7] have shown, however, that $E^{b}(k)$ displays two power-law ranges. Several authors 8 [12] have suggested that, to obtain these two power-law regimes, we must augment the MHD equations with a Hall-effect term, which leads to a scale separation at the ion-inertial length $d_{I}$ or, equivalently, at the wave number $k_{I}=2 \pi / d_{I}$. For $k<k_{I}, E^{b}(k) \propto k^{-\alpha^{b, 1}}$, it has been observed that $\alpha^{b, 1} \simeq 5 / 3$. For $k_{d}>k>k_{I}, E^{b}(k) \propto k^{-\alpha^{b, 2}}$, where $\alpha^{b, 2}$ is either $\simeq 7 / 3$ or $\simeq 11 / 3$. The value of $\alpha^{b, 2}$ depends on whether the magnetic energy dominates over the fluid kinetic energy, which occurs in the electron-
MHD (EMHD) 13 limit, or the converse, i.e., the ionMHD (IMHD) limit. These limits follow from the 3D Hall-MHD equations: EMHD is obtained if the induction term is sub-dominant to the Hall term; in the IMHD case these two terms are comparable to each other. In the EMHD limit, we obtain a single, characteristic scale and K41 phenomenology yields $\alpha^{b, 2}=7 / 3$; in the IMHD case a comparison of the transfer time, from the Hall-term, and a second time, from the induction part, followed by simple dimensional analysis yields $\alpha^{b, 2}=11 / 3$ [10, 12].

Direct numerical simulations (DNSs) 9 11] have just begun to resolve these two scaling ranges; but their spatial resolution is much more limited than has been achieved in DNS studies of MHD turbulence [3, 4]. Thus, they have not been used to study the scaling or multiscaling properties of order $p$ fluid and magnetic structure functions (defined below). However, measurements of such equal-time magnetic structure functions in solarwind measurements [7] show that, although there is significant multiscaling in the low- $k\left(k<k_{I}\right)$, power-law range of $E^{b}(k)$, the scaling exponents in the second, high$k\left(k_{d}>k>k_{I}\right)$ power-law range increase linearly with the order $p$. Thus, it behooves us to develop a theoretical understanding of these important and intriguing observations and to test them.

We show that a shell-model version of the 3D HallMHD equations [11, 12, which is a generalization of MHD shell models 14, 15, provides a natural theoretical model for investigating such multiscaling behaviors in structure functions in 3D Hall-MHD turbulence. Given the large range of scales that we can cover in this shell model [16], its magnetic spectrum $E^{b}(k)$ reveals two, distinct, power-law ranges. We carry out the most comprehensive numerical study of this 3D Hall-MHD shell model attempted so far; and thereby we characterize and quantify, for the first time, the properties of the order- $p$ magnetic and velocity structure functions in this model via 


\begin{tabular}{|l|c|c|c|c|c|c|c|c|}
\hline Runs & $\operatorname{Pr}_{M}$ & $\nu$ & $\nu_{2}$ & $\eta_{2}$ & $d_{I}$ & $P r_{M \text { eff }}$ & $\nu_{\text {eff }}\left(\times 10^{-6}\right)$ & $\eta_{\text {eff }}\left(\times 10^{-6}\right)$ \\
\hline R1 & 1 & $10^{-8}$ & $5 \times 10^{-13}$ & $5 \times 10^{-13}$ & 0.1 & $1.2 \pm 0.3$ & $1.8 \pm 0.2$ & $1.6 \pm 0.4$ \\
\hline R2 & 10 & $10^{-7}$ & $5 \times 10^{-12}$ & $5 \times 10^{-13}$ & 0.1 & $4.3 \pm 0.8$ & $4.7 \pm 0.4$ & $1.1 \pm 0.2$ \\
\hline R3 & 10 & $10^{-8}$ & $1 \times 10^{-13}$ & $1 \times 10^{-14}$ & 0.01 & $2.8 \pm 0.6$ & $0.74 \pm 0.09$ & $0.27 \pm 0.06$ \\
\hline R4 & 1 & $10^{-9}$ & $1 \times 10^{-14}$ & $1 \times 10^{-14}$ & 0.01 & $1.0 \pm 0.2$ & $0.31 \pm 0.04$ & $0.31 \pm 0.07$ \\
\hline \hline Runs & $\tau$ & $l_{I}$ & $R e_{\lambda}\left(\times 10^{5}\right)$ & $\eta_{d}^{u}\left(\times 10^{-4}\right)$ & $\eta_{d}^{b}\left(\times 10^{-4}\right)$ & $\alpha^{u}$ & $\alpha^{b, 1}$ & $\alpha^{b, 2}$ \\
\hline R1 & $16.3 \pm 0.6$ & $6.64 \pm 0.09$ & $11 \pm 2$ & $1.69 \pm 0.04$ & $2.2 \pm 0.3$ & $1.66 \pm 0.03$ & $1.75 \pm 0.03$ & $3.39 \pm 0.08$ \\
\hline R2 & $16 \pm 1$ & $6.7 \pm 0.1$ & $4.9 \pm 0.8$ & $3.40 \pm 0.08$ & $1.7 \pm 0.2$ & $1.65 \pm 0.03$ & $1.69 \pm 0.03$ & $3.45 \pm 0.06$ \\
\hline R3 & $16 \pm 1$ & $6.6 \pm 0.1$ & $26 \pm 4$ & $0.90 \pm 0.03$ & $0.62 \pm 0.07$ & $1.69 \pm 0.03$ & $1.74 \pm 0.02$ & $3.34 \pm 0.09$ \\
\hline R4 & $13.3 \pm 0.7$ & $6.8 \pm 0.1$ & $64 \pm 9$ & $0.46 \pm 0.02$ & $0.64 \pm 0.07$ & $1.70 \pm 0.02$ & $1.74 \pm 0.02$ & $3.28 \pm 0.08$ \\
\hline
\end{tabular}

TABLE I: The values of the different parameters (see text) used in our runs R1 - R4 and the spectral exponents $\alpha^{u}, \alpha^{b, 1}$, and $\alpha^{b, 2}$ obtained.

their scaling exponents $\zeta_{p}^{u}$ (fluid), $\zeta_{p}^{b, 1}$ (magnetic, $k<k_{I}$ regime), and $\zeta_{p}^{b, 2}$ (magnetic, $k_{d}>k>k_{I}$ regime). We find that all three sets of exponents show clear signatures of multiscaling. In particular, we find the remarkable result that magnetic structure functions display multiscaling for both the low- $k$ and the high- $k$ power-law ranges. A second significant and surprising finding is that, although the exponents $\zeta_{p}^{b, 2} \neq \zeta_{p}^{b, 1}, \zeta_{p}^{b, 2} \neq \zeta_{p}^{u}$, the exponent ratios $\frac{\zeta_{p}^{b, 2}}{\zeta_{3}^{b, 2}} \simeq \frac{\zeta_{p}^{b, 1}}{\zeta_{3}^{b, 1}} \simeq \frac{\zeta_{p}^{u}}{\zeta_{3}^{u}}[17$.

The 3D Hall-MHD equations for the velocity $\mathbf{u}$ and magnetic $\mathbf{b}$ fields are

$$
\begin{aligned}
\frac{\partial \mathbf{u}}{\partial t}+(\mathbf{u} \cdot \nabla) \mathbf{u} & =-\nabla p+\mathbf{j} \times \mathbf{b}+\nu \nabla^{2} \mathbf{u} \\
\frac{\partial \mathbf{b}}{\partial t} & =\nabla \times\left[\left(\mathbf{u}-d_{I} \mathbf{j}\right) \times \mathbf{b}\right]+\eta \nabla^{2} \mathbf{b}
\end{aligned}
$$

here $\nu$ and $\eta$ are the kinematic viscosity and magnetic diffusivity, respectively, $d_{I}$ is the ion-inertial length, the scale at which the Hall effect becomes important, the current density vector $\mathbf{j}=\nabla \times \mathbf{b}$, the pressure is $p, \nabla \cdot \mathbf{b}=\mathbf{0}$, and, at low Mach numbers, the flow is incompressible, i.e., $\nabla \cdot \mathbf{u}=\mathbf{0}$. We define the dissipation length scales $\eta_{d}^{u}=\left(\nu^{3} / \varepsilon^{u}\right)^{1 / 4}$ and $\eta_{d}^{b}=\left(\eta^{3} / \varepsilon^{b}\right)^{1 / 4}$, where $\varepsilon^{u}$ and $\varepsilon^{b}$ are the kinetic and magnetic-energy dissipation rates, respectively; we restrict ourselves to decaying turbulence, so we do not include forcing terms. The Hall term, which is a singular perturbation of the MHD equations [10, has a significant effect if $d_{I} \gg \eta_{d}^{u}, \eta_{d}^{b}$. The shell-model versions of Eq. (1) are [11, 12]:

$$
\begin{aligned}
& \frac{d u_{n}}{d t}=-\nu k_{n}^{2} u_{n}-\nu_{2} k_{n}^{4} u_{n}+\iota\left[\Phi_{n}^{u}\right]^{*}, \\
& \frac{d b_{n}}{d t}=-\eta k_{n}^{2} b_{n}-\eta_{2} k_{n}^{4} b_{n}+\iota\left[\Phi_{n}^{b}\right]^{*},
\end{aligned}
$$

where $u_{n}$ and $b_{n}$ are, respectively, the complex velocity and magnetic field in the shell $n, *$ denotes complex conjugation, $1 \leq n \leq N$, where $N$ is the total number of shells, $\Phi_{n}^{u}=A_{n}\left(u_{n+1} u_{n+2}-b_{n+1} b_{n+2}\right)+$
$B_{n}\left(u_{n-1} u_{n+1}-b_{n-1} b_{n+1}\right)+C_{n}\left(u_{n-2} u_{n-1}-b_{n-2} b_{n-1}\right)$ and $\Phi_{n}^{b}=D_{n}\left(u_{n+1} b_{n+2}-b_{n+1} u_{n+2}\right)+E_{n}\left(u_{n-1} b_{n+1}-\right.$ $\left.b_{n-1} u_{n+1}\right)-F_{n}\left(u_{n-2} b_{n-1}-b_{n-2} u_{n-1}\right)-d_{I}\left[G_{n} b_{n+1} b_{n+2}+\right.$ $\left.H_{n} b_{n-1} b_{n+1}+I_{n} b_{n-2} b_{n-1}\right]$, with $A_{n}=k_{n}$, $B_{n}=-\frac{1}{2} k_{n-1}, C_{n}=-\frac{1}{2} k_{n-2}, D_{n}=\frac{1}{6} k_{n}, E_{n}=\frac{1}{3} k_{n-1}$, $F_{n}=\frac{2}{3} k_{n-2}, G_{n}=-\frac{1}{2}(-1)^{n} k_{n}^{2}, H_{n}=-\frac{1}{2}(-1)^{n-1} k_{n-1}^{2}$, $I_{n}=(-1)^{n-2} k_{n-2}^{2}, k_{n}=2^{n} k_{0}$, and $k_{0}=1 / 16$; the values of the coefficients $A_{n}-I_{n}$ are determined by enforcing the shell-model analogs of the Hall-MHD conservation laws, in the inviscid, unforced limit; the conserved quantities are the total energy $E=\Sigma_{n}\left(\left|u_{n}\right|^{2}+\left|b_{n}\right|^{2}\right) / 2$, the magnetic helicity $H_{M}=\Sigma_{n}(-1)^{n}\left|b_{n}\right|^{2} / 2 k_{n}$, and the ion helicity $H_{I}=\Sigma_{n}\left(\left(b_{n} u_{n}^{*}+b_{n}^{*} u_{n}\right)+d_{I}(-1)^{n} k_{n}\left|u_{n}\right|^{2} / 2\right)$; the hyperviscosity $\nu_{2}$ and the magnetic hyperdiffusivity $\eta_{2}$ have to be included for numerical stability [10, 12]. We use the boundary conditions $A_{N-1}=A_{N}=B_{1}=B_{N}=C_{1}=C_{2}=0$, $D_{N-1}=D_{N}=E_{1}=E_{N}=F_{1}=F_{2}=0$, $G_{N-1}=G_{N}=H_{1}=H_{N}=I_{1}=I_{2}=0$, and the following initial values for $u_{n}=u^{(0)} k_{n}^{-1 / 3} e^{-k_{n}^{2}+\iota \phi^{u}}$ and $b_{n}=b^{(0)} k_{n}^{-1 / 3} e^{-k_{n}^{2}+\iota \phi^{b}}$; here $u^{(0)}=0.5, b^{(0)}=0.05$, and the random phases $\phi^{u}$ and $\phi^{b}$ are distributed uniformly on the interval $[-\pi,+\pi]$; different values of these random phases distinguish different initial conditions; we work with decaying turbulence, so there is no forcing term; and our results are averaged over 7500 independent initial conditions [18. We set $N=22$, use a secondorder, slaved Adams-Bashforth scheme [20] for solving the shell-model ordinary differential equations (2), and calculate the energy spectra $E^{u}\left(k_{n}\right)=\frac{1}{2}\left|u_{n}\right|^{2} / k_{n}$ and $E^{b}\left(k_{n}\right)=\frac{1}{2}\left|b_{n}\right|^{2} / k_{n}$ (the superscripts $u$ and $b$ refer to velocity and magnetic field, respectively), the rootmean-square velocity $u_{r m s}=\sqrt{\Sigma_{n}\left|u_{n}\right|^{2}}$, the Taylor microscale $\lambda=\sqrt{\Sigma_{n} E^{u}\left(k_{n}\right) / \Sigma_{n} k_{n}^{2} E^{u}\left(k_{n}\right)}$, the Taylormicroscale Reynolds number $R e_{\lambda}=u_{r m s} \lambda / \nu_{\text {eff }}$, the integral length scale $l_{I}=\Sigma_{n}\left(E^{u}\left(k_{n}\right) / k_{n}\right) / \Sigma_{n} E^{u}\left(k_{n}\right)$, the effective viscosity and magnetic diffusivity $\nu_{\text {eff }}=\Sigma_{n}\left(\nu k_{n}^{2} E^{u}\left(k_{n}\right)+\nu_{2} k_{n}^{4} E^{u}\left(k_{n}\right)\right) / \Sigma_{n} k_{n}^{2} E^{u}\left(k_{n}\right)$ and $\eta_{\mathrm{eff}}=\Sigma_{n}\left(\eta k_{n}^{2} E^{b}\left(k_{n}\right)+\eta_{2} k_{n}^{4} E^{b}\left(k_{n}\right)\right) / \Sigma_{n} k_{n}^{2} E^{b}\left(k_{n}\right)$, 

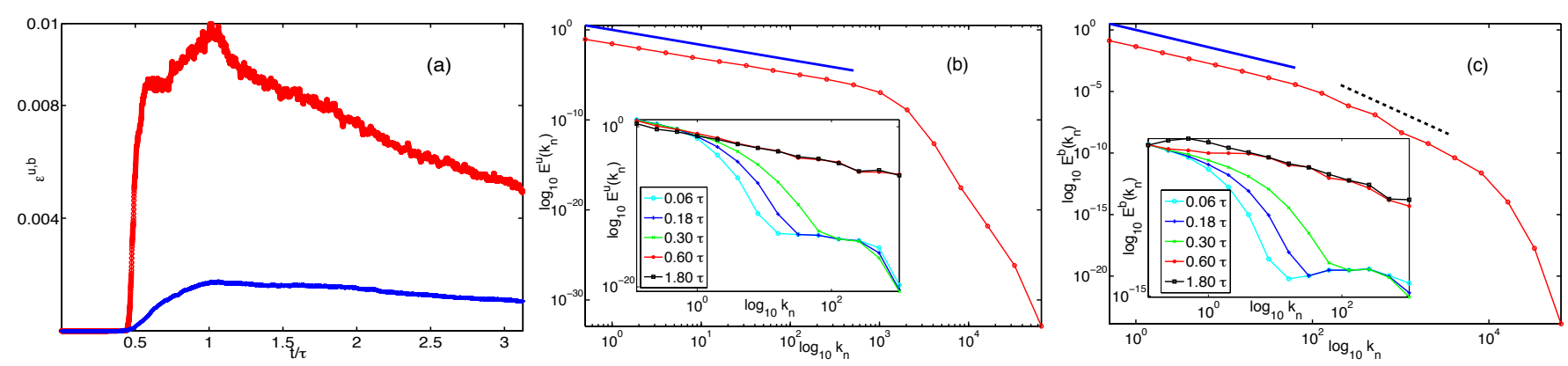

FIG. 1: (Color online) (a) Plots of $\varepsilon^{u}$ (red, upper curve) and $\varepsilon^{b}$ (blue, lower curve) versus the rescaled time $t / \tau$. (b) Plot of the kinetic-energy spectrum $E^{u}(k)$; the thick, blue line indicates the K41-scaling power law. Inset : Time evolution of $E^{u}(k)$ during the initial period with transients; the legend indicates the time at which each curve is obtained. (c) Plot of the magnetic-energy spectrum $E^{b}(k)$; the thick, blue line indicates K41 scaling in the low- $k$ region and the dashed, black line indicates $k^{-3.5}$ scaling in the high- $k$ region. Inset : Time evolution of $E^{b}(k)$ during the initial period with transients; the legend indicates the time at which each curve is obtained. Here we use $E^{u}\left(k_{n}\right)=\Sigma_{2}^{u}\left(k_{n}\right) / k_{n}$ and $E^{b}\left(k_{n}\right)=\Sigma_{2}^{b}\left(k_{n}\right) / k_{n}$ to suppress 3 -cycles (see text). The data shown are from run R2 and the spectral exponents for all our runs are reported in Table I similar plots for runs R1, R3, and R4 are given in the Supplemental Material [21].

respectively, the effective magnetic Prandtl number 22] $P r_{M \text { eff }}=\nu_{\text {eff }} / \eta_{\text {eff }}$, and the dissipation rates $\varepsilon^{u}=\nu_{\text {eff }} \Sigma_{n} k_{n}^{2} E^{u}\left(k_{n}\right)$ and $\varepsilon^{b}=\eta_{\text {eff }} \Sigma_{n} k_{n}^{2} E^{b}\left(k_{n}\right)$. The parameters of our simulations are given in Table I.

In shell models, the equal-time, order- $p$ structure functions for the velocity field and the magnetic field are defined, respectively, as $S_{p}^{u}\left(k_{n}\right)=\left\langle\left|u_{n}\right|^{p}\right\rangle \sim k_{n}^{\zeta_{p}^{u}}$ and $S_{p}^{b}\left(k_{n}\right)=\left\langle\left|b_{n}\right|^{p}\right\rangle$, where $S_{p}^{b}\left(k_{n}\right) \sim k_{n}^{\zeta_{p}^{b, 1}}\left(k<k_{I}\right)$ and $S_{p}^{b}\left(k_{n}\right) \sim k_{n}^{\zeta_{p}^{b, 2}}\left(k_{d}>k>k_{I}\right)$. However, to remove the effects of an underlying three cycle in GOY-type shell models 14, 16, we use the modified structure functions $\Sigma_{p}^{u}\left(k_{n}\right)=\left\langle\left|\Im\left[u_{n+2} u_{n+1} u_{n}+1 / 4 u_{n-1} u_{n} u_{n+1}\right]\right|^{p / 3}\right\rangle$ and $\Sigma_{p}^{b}\left(k_{n}\right)=\left\langle\left|\Im\left[b_{n+2} b_{n+1} b_{n}+1 / 4 b_{n-1} b_{n} b_{n+1}\right]\right|^{p / 3}\right\rangle$, from which we can obtain multiscaling exponents via $\Sigma_{p}^{u}\left(k_{n}\right) \sim$ $k_{n}^{\zeta_{p}^{u}}, \Sigma_{p}^{b}\left(k_{n}\right) \sim k_{n}^{\zeta_{p}^{b, 1}}\left(k_{n}<k_{I}\right)$, and $\Sigma_{p}^{b}\left(k_{n}\right) \sim k_{n}^{\zeta_{p}^{b, 2}}$ $\left(k_{d}>k_{n}>k_{I}\right)$. We also use the extended self-similarity (ESS) procedure 23. to determine exponent ratios from slopes of $\log$-log plots of $\Sigma_{p}^{u}$ versus $\Sigma_{3}^{u}$ and their magnetic counterparts (Fig. 2 inset).

In Fig. 1(a) we show plots of $\varepsilon^{u}$ (red, upper curve) and $\varepsilon^{b}$ (blue, lower curve) versus the rescaled time $t / \tau$, for run R2, where the box-size eddy-turnover time $\tau=1 /\left(u_{1} k_{1}\right)$ is evaluated at the principal peak of $\varepsilon^{u}$. This peak signals the completion of the Richardson cascade 3], as we can see from the time evolution of $E^{u}\left(k_{n}\right)$ and $E^{b}\left(k_{n}\right)$, in the insets of Figs.1(b) and (c), respectively, where the red lines with full circles denote the spectra at cascade completion. We evaluate the spectral-slope exponent $\alpha^{u}$ at cascade completion from log-log plots of $E^{u}\left(k_{n}\right)$ versus $k_{n}$ as shown in Fig. 1(b). We find $\alpha^{u} \simeq 5 / 3$, as predicted by dimensional analysis, and illustrated in Fig. 1(b) by a thick, blue line. In Fig. 1(c) we show a representative plot of $E^{b}\left(k_{n}\right)$; we see two different scaling regimes clearly: (1) from the low- $k$ one (solid, blue line), we find that $\alpha^{b, 1} \simeq 5 / 3$, which is consistent with dimen-

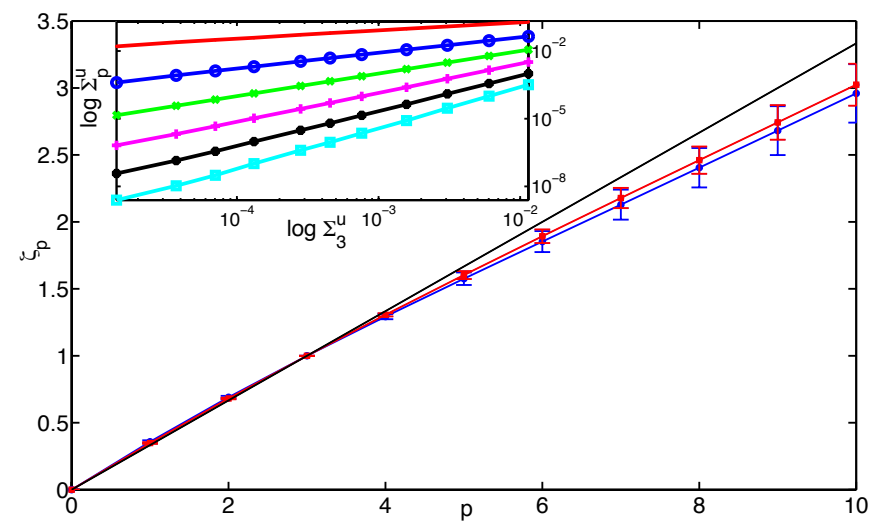

FIG. 2: (Color online) The exponents $\zeta_{p}^{u}$ (blue filled circles connected by a blue line), $\zeta_{p}^{b, 1}$ (red filled squares connected by a red line), and the K41 prediction (thick black line) versus $p$. The lines connecting the data points are a guide to the eye. (Inset) A representative plot of $\Sigma_{p}^{u}$ versus $\Sigma_{3}^{u}$; equaltime exponents are calculated from such plots. The different curves are for $p=1$ (top) ...6 (bottom) and are from run R2.

sional analysis; (2) from the high- $k$ regime, we obtain $\alpha^{b, 2}=3.45 \pm 0.06$, which is close to the dimensionalanalysis value $11 / 3$ for IMHD systems, such as ours, in which induction and Hall terms are comparable [10, 12. These spectral exponents are consistent with those in solar-wind experiments [7. In Table I we provide our results for all three spectral exponents; we obtain the values of these and all other exponents from the means of our runs with 7500 independent initial conditions; the error bars follow from the associated standard deviations.

To characterize the statistical properties of the HallMHD system, we now calculate the equal-time exponents $\zeta_{p}^{u}, \zeta_{p}^{b, 1}\left(k<k_{I}\right)$, and $\zeta_{p}^{b, 2}\left(k_{d}>k>k_{I}\right)$ via the $\bmod -$ ified structure functions $\Sigma_{p}^{u}$ and $\Sigma_{p}^{b}$, just after cascade 


\begin{tabular}{|c|c|c|c|c|}
\hline$p$ & $\zeta_{p}^{u}$ & $\zeta_{p}^{b, 1}$ & $\zeta_{p}^{b, 2}$ & $\zeta_{p}^{b, 2} / \zeta_{3}^{b, 2}$ \\
\hline 1 & $0.36 \pm 0.01$ & $0.348 \pm 0.006$ & $1.40 \pm 0.04$ & $0.38 \pm 0.01$ \\
2 & $0.69 \pm 0.01$ & $0.682 \pm 0.006$ & $2.6 \pm 0.1$ & $0.698 \pm 0.009$ \\
3 & 1.0 & 1.0 & $3.7 \pm 0.2$ & 1.0 \\
4 & $1.29 \pm 0.02$ & $1.31 \pm 0.01$ & $4.8 \pm 0.3$ & $1.30 \pm 0.01$ \\
5 & $1.57 \pm 0.06$ & $1.60 \pm 0.03$ & $6.0 \pm 0.4$ & $1.60 \pm 0.03$ \\
6 & $1.85 \pm 0.09$ & $1.89 \pm 0.05$ & $7.1 \pm 0.4$ & $1.90 \pm 0.04$ \\
7 & $2.1 \pm 0.1$ & $2.18 \pm 0.08$ & $8.2 \pm 0.6$ & $2.20 \pm 0.06$ \\
8 & $2.4 \pm 0.2$ & $2.5 \pm 0.1$ & $9.3 \pm 0.6$ & $2.50 \pm 0.07$ \\
9 & $2.7 \pm 0.2$ & $2.7 \pm 0.1$ & $10.5 \pm 0.7$ & $2.81 \pm 0.08$ \\
10 & $3.0 \pm 0.3$ & $3.0 \pm 0.2$ & $11.6 \pm 0.8$ & $3.11 \pm 0.09$ \\
\hline
\end{tabular}

TABLE II: Multiscaling exponents $\zeta_{p}^{u}, \zeta_{p}^{b, 1}, \zeta_{p}^{b, 2}$, and the exponent ratio $\zeta_{p}^{b, 2} / \zeta_{3}^{b, 2}$ for run $\mathrm{R} 2$. The exponents from the other runs are equal to the ones shown here. We note that $\zeta_{p}^{u} \simeq \zeta_{p}^{b, 1} \simeq \zeta_{p}^{b, 2} / \zeta_{3}^{b, 2} \neq \zeta_{p}^{b, 2}[21$.

completion. We find $\zeta_{3}^{u}=1=\zeta_{3}^{b, 1}$, which is consistent with dimensional analysis. In Fig. 2 we show the order- $p$ equal-time exponents $\zeta_{p}^{u}$ (blue, filled circles) and $\zeta_{p}^{b, 1}$ (red, filled squares) for integer values of $p$ between 1 and 10; the thick, black line illustrates the dimensional, simple K41 scaling. We see that $\zeta_{p}^{u} \simeq \zeta_{p}^{b, 1}$ and both exponents show clear multiscaling corrections to K41 scaling, with values consistent with those obtained in 3D MHD turbulence 3. We obtain these multiscaling exponents by using $\Sigma_{p}^{u}$ and $\Sigma_{p}^{b}$ and the ESS procedure [23], to extend the scaling range. However, the result $\zeta_{3}^{u} \simeq 1$ ensures that the exponent ratios and the exponents themselves are equal (within error bars). In Table II, we list the order- $p$ equal-time exponents $\zeta_{p}^{u}$ and $\zeta_{p}^{b, 1}$ for integer values of $p$ between 1 and 10 .

We finally turn to the exponents $\zeta_{p}^{b, 2}$, which characterize the high- $k$ regime $\left(k_{d}>k>k_{I}\right)$. Solar-wind measurements [7] of $\zeta_{p}^{b, 2}$ suggest simple-scaling behaviour, with $\zeta_{p}^{b, 2}$ a linear function of $p$. In Fig. 3 we plot $\zeta_{p}^{b, 2}$ (obtained without ESS) versus $p$. Our results for $\zeta_{p}^{b, 2}$ are in qualitative agreement with solar-wind measurements to the extent that there is only mild multiscaling; i.e., $\zeta_{p}^{b, 2}$ is a nonlinear, monotone, increasing function of $p$, but the deviation from a linear dependence on $p$ is not very pronounced. Although the exponents $\zeta_{p}^{b, 2}$, for all the runs R1-R4, are in agreement with each other, given our error-bars, their mean values seem to decrease with $d_{I}$. We now use the ESS procedure to obtain the exponent ratios $\zeta_{p}^{b, 2} / \zeta_{3}^{b, 2}$, which are plotted versus $p$ in the inset of Fig. 3 (note $\zeta_{3}^{b, 2} \neq 1$ ). This ESS plot is remarkable for two reasons : (1) There is a clear signature of multiscaling (the thick, black line in the inset indicates simple scaling); (2) although the exponents $\zeta_{p}^{b, 2}$ are very different from $\zeta_{p}^{u}$ and $\zeta_{p}^{b, 1}$, the ratios $\zeta_{p}^{b, 2} / \zeta_{3}^{b, 2}$ are equal to $\zeta_{p}^{u}$ and $\zeta_{p}^{b, 1}$ (within error bars). In Table II, we list

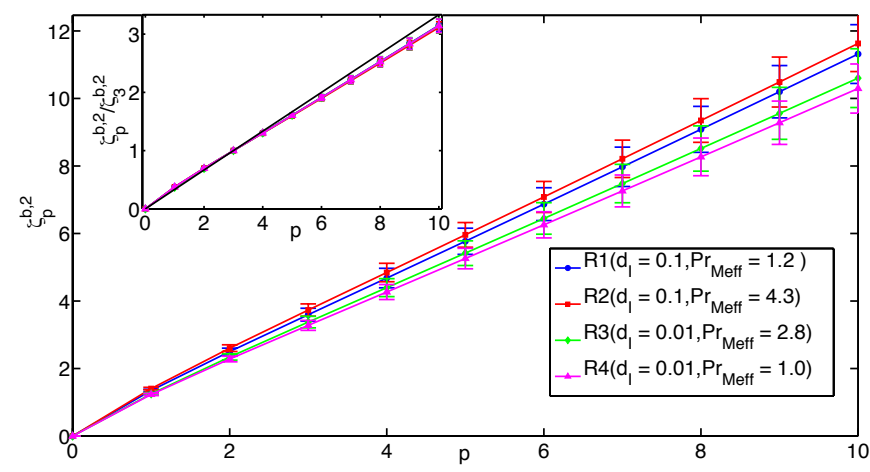

FIG. 3: (Color online) Plot of the exponents $\zeta_{p}^{b, 2}$ versus the order $p$ showing a mild departure from linear scaling. Inset : the exponent ratios $\zeta_{p}^{b, 2} / \zeta_{3}^{b, 2}$ (obtained via ESS) versus $p$, showing clear multiscaling. The thick, black line indicates simple scaling. The Exponents $\zeta_{p}^{b, 2}$ and $\zeta_{p}^{b, 2} / \zeta_{3}^{b, 2}$ are shown for runs R1-R4 (see legend).

both $\zeta_{p}^{b, 2}$ and $\zeta_{p}^{b, 2} / \zeta_{3}^{b, 2}$ for different values of $p$ for the representative run $\mathrm{R} 2$.

We hope our extensive studies of the multiscaling of structure functions in a shell model for 3D Hall-MHD will stimulate high-precision and high-resolution experimental and DNS studies to determine conclusively whether 3D Hall-MHD turbulence shows multiscaling for $k_{d}>$ $k>k_{I}$. Our ESS results suggest that structure functions show mild, but distinct, multiscaling in this region To obtain quantitative agreement with solar-wind exponents, we must, of course, carry out DNS studies of the 3D Hall-MHD equations (1) and include compressibility effects and a mean magnetic field [8-10]; however, current computational resources limit severely the spatial resolution of such DNS studies so they cannot (a) uncover the multiscaling of magnetic-field structure functions in $3 \mathrm{D}$ Hall-MHD turbulence in both low- and high- $k$ power-law ranges and (b) obtain well-averaged multiscaling exponent ratios. For the moment, therefore, the shell-model study, which we have undertaken, provides the only way of understanding the multiscaling of structure functions in the solar wind [7] and the apparent and intriguing universality of the exponent ratios. This apparant universality needs to be investigated in detail in experiments and DNS.

We thank A. Basu and V. Krishan for discussions, S. Galtier for the preprint of Ref. 10, CSIR, UGC, and DST (India) for support, and SERC (IISc) for computational resources. R.P. and G.S. are members of the International Collaboration for Turbulence Research; G.S., R.P., and S.S.R. acknowledge support from the COST Action MP0806; S.S.R. thanks the European Research Council for support under the European Communitys Seventh Framework Program (FP7/2007-2013, Grant Agreement no. 240579). 
* Electronic address: debarghya@physics.iisc.ernet.in

† Electronic address: samriddhisankarray@gmail.com Formerly at Laboratoire Lagrange, OCA, UNS, CNRS, BP 4229, 06304 Nice Cedex 4, France

‡ Electronic address: ganapati.sahoo@ds.mpg.de

$\S$ Electronic address: rahul@physics.iisc.ernet.in Also at Jawaharlal Nehru Centre For Advanced Scientific Research, Jakkur, Bangalore, India

[1] A. R. Choudhuri, The Physics of Fluids and Plasmas: An Introduction for Astrophysicists (Cambridge University Press, Cambridge, UK, 1998); V. Krishan, Astrophysical Plasmas and Fluids (Kluwer, Dordrecht, (1999); D. Biskamp, Magnetohydrodynamic Turbulence (Cambridge University Press, Cambridge, UK, 2003) ; G. Rüdiger and R. Hollerbach, The Magnetic Universe: Geophysical and Astrophysical Dynamo Theory (Wiley, Weinheim, 2004); M.K. Verma, Phys. Rep. 401, 229 (2004).

[2] S. Cowley, J.-F. Pinton, and A. Pouquet, eds. New J. Phys. 9 (2007).

[3] G. Sahoo, P. Perlekar and R. Pandit, New J. Phys. 13, 0130363 (2011).

[4] D. Biskamp and W.-C. Müller Phys. Plasmas, 7, 4889 (2008); P.D. Mininni and A. Pouquet Phys. Rev. E 80, 025401 (2009); A. Brandenburg, D. Sokoloff, and K. Subramanian, Space Science Reviews, 169, Issue 1-4, 123 (2012).

[5] A.N. Kolmogorov, Dokl. Akad. Nauk. SSSR 30, 299303 (1941).

[6] U. Frisch, Turbulence: the Legacy of A.N. Kolmogorov, (Cambridge University Press, Cambridge, UK, 1995).

[7] K.H. Kiyani, et al., Phys. Rev. Lett. 103, 075006 (2009); C.W. Smith, K. Hamilton, B.J. Vasquez and R.J. Leamon, The Astrophysical Journal 645, L85 (2006).

[8] V. Krishan and S.M. Mahajan, Solar Physics 22029 (2004); J. Geophys. Research 109, A11105 (2004).

[9] D. Shaikh and P.K. Shukla, Phys. Rev. Lett. 102, 045004 (2009); P.D. Mininni, A. Alexakis, and A. Pouquet, J. Plasma Phys., 73, Part 3, 377 (2007).

[10] R. Meyrand and S. Galtier, Phys. Rev. Lett. 109, 194501 (2012).

[11] D. Hori, M. Furukawa, S. Ohsaki, and Z. Yoshida, J. Plasma Fusion Res. 81 No.3, 141 (2005); D. Hori and H. Miura, Plasma and Fusion Research 3, s1053 (2008).

[12] S. Galtier, and E. Buchlin The Astrophysical Journal 656, 560 (2007).

[13] D. Biskamp, E. Schwarz, and J.F. Drake, Phys. Rev. Lett. 76, 1264 (1996).

[14] A. Basu, A. Sain, S. Dhar, and R. Pandit, Phys. Rev. Lett. 81, 2687 (1998); C. Kalelkar and R. Pandit, Phys. Rev. E 69, 046304 (2004); G. Sahoo, D. Mitra, and R. Pandit, Phys. Rev. E 81, 036317 (2010).

[15] P. Frick and D. Sokoloff, Phys. Rev. E 57, 4155 (1998); S.A. Lozhkin, D.D. Sokolov, and P.G. Frick, Astron. Rep. 43, 753 (1999); A. Brandenburg, K. Enqvist, and P. Olesen, Phys. Rev. D 54, 1291 (1996); P. Giuliani and V. Carbone, Europhys. Lett. 533(5), 527 (1998).

[16] E. Gledzer, Sov. Phys. Dokl. 18, 216 (1973); K. Ohkitani and M. Yamada, Prog. Theor. Phys. 81, 329 (1989); L.P. Kadanoff, D. Lohse, and J. Wang, Phys. Fluids 7, 517 (1995).
[17] We note, in passing, that $\zeta_{p}^{u} \simeq \zeta_{p}^{b, 1} \simeq \zeta_{p}^{\mathrm{fl}}$, where $\zeta_{p}^{\mathrm{fl}}$ are the equal-time scaling exponents obtained for $3 \mathrm{D}$ fluid turbulence [6].

[18] Earlier studies have suggested the strong universality of scaling exponents, i.e., the equality of multiscaling exponents obtained from studies of decaying and forced turbulence [19].

[19] V.S. L'vov, R. A. Pasmanter, A. Pomyalov, and I. Procaccia, Phys. Rev. E 67066310 (2003); S. S. Ray, D. Mitra, and R. Pandit, New. J. Phys 10, 033003 (2008).

[20] S. M. Cox, and P. C. Matthews J. Sci. Computing 176, 430 (2002).

[21] See Supplemental Material at [URL to be inserted by publisher] for analogous results from runs R1, R3, and R4.

[22] A.B. Iskakov, et al., Phys. Rev. Lett. 98, 208501 (2007).

[23] R. Benzi, S. Ciliberto, C. Baudet, F. Massaioli and S. Succi, Phys. Rev. E 48, R29 (1993); S. Chakraborty, U. Frisch and S.S. Ray, J. Fluid Mech. 649, 275 (2010).

\section{SUPPLEMENTAL MATERIAL}

In this Supplemental Material we describe details of our work that are of interest only to specialists of the field. The notations and abbreviations used in this Supplemental Material are the same as in the main paper.

In our main paper, we discuss the statistical nature of turbulence in the Hall-MHD plasma and, in particular, the scaling properties of various structure functions, in great detail. To substantiate our claims, we show in the main paper representative data from only a single set of simulations (except in Fig. 3 where we show results from all our simulations), namely, Run R2 (see Table I of the main paper). In Fig. 3 of the main paper we do show exponents from all the four different sets of simulation (as detailed in Table I of the main paper); however, in Table II (main paper) we list exponents from R2 only; because the different sets of simulations all agree with each other, representative data from one set of simulations (R2), in the main paper, is enough to highlight the nature of multiscaling in Hall-MHD turbulence.

We give here the equal-time exponents from the runs R1, R3, R4 in Table I; these exponents are in agreement with the ones listed for R2 in Table II of the main paper. Furthermore, we show plots for the fluid and magnetic energy dissipation rates (Fig 1(a) for run R1, Fig 2(a) for run R3, and Fig 3(a) for run R4), the kinetic energy spectrum (Fig 1(b) for run R1, Fig 2(b) for run R3, and Fig 3(b) for run R4), and the magnetic energy spectrum (Fig 1(c) for run R1, Fig 2(c) for run R3, and Fig 3(c) for run R4); these plots are analogous to the plots shown in Fig.1, for run R2, in the main paper.

The parameters of the runs R1, R3, and R4 (along with those for run R2) are given in Table (I) of our main paper. 


\begin{tabular}{|c|c|c|c|c|c|c|c|c|c|}
\hline & \multicolumn{3}{|c|}{$\mathrm{R} 1$} & \multicolumn{3}{|c|}{ R3 } & \multicolumn{3}{|c|}{$\mathrm{R} 4$} \\
\hline$p$ & $\zeta_{p}^{u} / \zeta_{3}^{u}$ & $\zeta_{p}^{b, 1} / \zeta_{3}^{b, 1}$ & $\zeta_{p}^{b, 2} / \zeta_{3}^{b, 2}$ & $\zeta_{p}^{u} / \zeta_{3}^{u}$ & $\zeta_{p}^{b, 1} / \zeta_{3}^{b, 1}$ & $\zeta_{p}^{b, 2} / \zeta_{3}^{b, 2}$ & $\zeta_{p}^{u} / \zeta_{3}^{u}$ & $\zeta_{p}^{b, 1} / \zeta_{3}^{b, 1}$ & $\zeta_{p}^{b, 2} / \zeta_{3}^{b, 2}$ \\
\hline 1 & $0.36 \pm 0.01$ & $0.349 \pm 0.007$ & $0.38 \pm 0.01$ & $0.36 \pm 0.01$ & $0.348 \pm 0.005$ & $0.37 \pm 0.01$ & $0.355 \pm 0.007$ & $0.349 \pm 0.005$ & $0.38 \pm 0.01$ \\
\hline 2 & $0.69 \pm 0.01$ & $0.682 \pm .007$ & $0.70 \pm .01$ & $0.69 \pm 0.01$ & $0.681 \pm .005$ & $0.69 \pm .01$ & $0.687 \pm 0.008$ & $0.682 \pm .005$ & $0.70 \pm .01$ \\
\hline 3 & 1 & 1 & 1 & 1 & 1 & 1 & 1 & 1 & 1 \\
\hline 4 & $1.29 \pm 0.02$ & $1.30 \pm 0.01$ & $1.30 \pm 0.01$ & $1.30 \pm 0.02$ & $1.306 \pm 0.009$ & $1.30 \pm 0.01$ & $1.30 \pm 0.02$ & $1.31 \pm 0.01$ & $1.30 \pm 0.01$ \\
\hline 5 & $1.57 \pm 0.06$ & $1.60 \pm 0.03$ & $1.61 \pm 0.03$ & $1.58 \pm 0.04$ & $1.60 \pm 0.02$ & $1.61 \pm .03$ & $1.58 \pm 0.04$ & $1.60 \pm 0.03$ & $1.60 \pm .03$ \\
\hline 6 & $1.8 \pm 0.1$ & $1.89 \pm 0.05$ & $1.91 \pm 0.04$ & $1.87 \pm 0.07$ & $1.89 \pm 0.04$ & $1.91 \pm 0.05$ & $1.87 \pm 0.06$ & $1.89 \pm 0.04$ & $1.91 \pm 0.05$ \\
\hline 7 & $2.1 \pm 0.1$ & $2.18 \pm 0.08$ & $2.22 \pm 0.06$ & $2.1 \pm 0.1$ & $2.18 \pm 0.05$ & $2.22 \pm 0.07$ & $2.15 \pm 0.08$ & $2.18 \pm 0.07$ & $2.22 \pm 0.06$ \\
\hline 8 & $2.4 \pm 0.2$ & $2.4 \pm 0.1$ & $2.53 \pm 0.07$ & $2.4 \pm 0.1$ & $2.46 \pm 0.07$ & $2.52 \pm 0.09$ & $2.4 \pm 0.1$ & $2.47 \pm 0.09$ & $2.52 \pm 0.08$ \\
\hline 9 & $2.7 \pm 0.2$ & $2.7 \pm 0.1$ & $2.84 \pm 0.09$ & $2.7 \pm 0.2$ & $2.74 \pm 0.09$ & $2.8 \pm 0.1$ & $2.7 \pm 0.1$ & $2.8 \pm 0.1$ & $2.8 \pm 0.1$ \\
\hline 10 & $3.0 \pm 0.2$ & $3.0 \pm 0.1$ & $3.2 \pm 0.1$ & $3.0 \pm 0.2$ & $3.0 \pm 0.1$ & $3.1 \pm 0.1$ & $3.0 \pm 0.2$ & $3.0 \pm 0.1$ & $3.1 \pm 0.1$ \\
\hline
\end{tabular}

TABLE III: Multiscaling exponent ratios $\zeta_{p}^{u} / \zeta_{3}^{u}, \zeta_{p}^{b, 1} / \zeta_{3}^{b, 1}$, and $\zeta_{p}^{b, 2} / \zeta_{3}^{b, 2}$ with error bars for the order $p$ in the range $1 \leq p \leq 10$ and runs R1, R3, and R4.
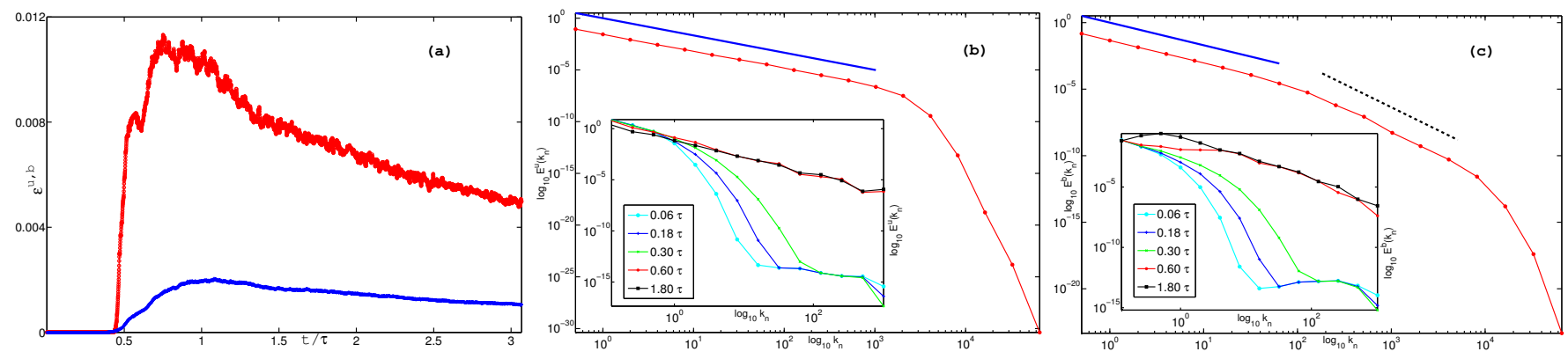

FIG. 4: (Color online) (a) Plots of $\varepsilon^{u}$ (red, upper curve) and $\varepsilon^{b}$ (blue, lower curve) versus the rescaled time $t / \tau$. (b) Plot of the kinetic-energy spectrum $E^{u}(k)$; the thick, blue line indicates the K41-scaling power law. Inset : Time evolution of $E^{u}(k)$ during the initial period with transients; the legend indicates the time at which each curve is obtained. (c) Plot of the magnetic-energy spectrum $E^{b}(k)$; the thick, blue line indicates K41 scaling in the low- $k$ region and the dashed, black line indicates $k^{-3.5}$ scaling in the high- $k$ region. Inset : Time evolution of $E^{b}(k)$ during the initial period with transients; the legend indicates the time at which each curve is obtained. The data shown are for run R1.
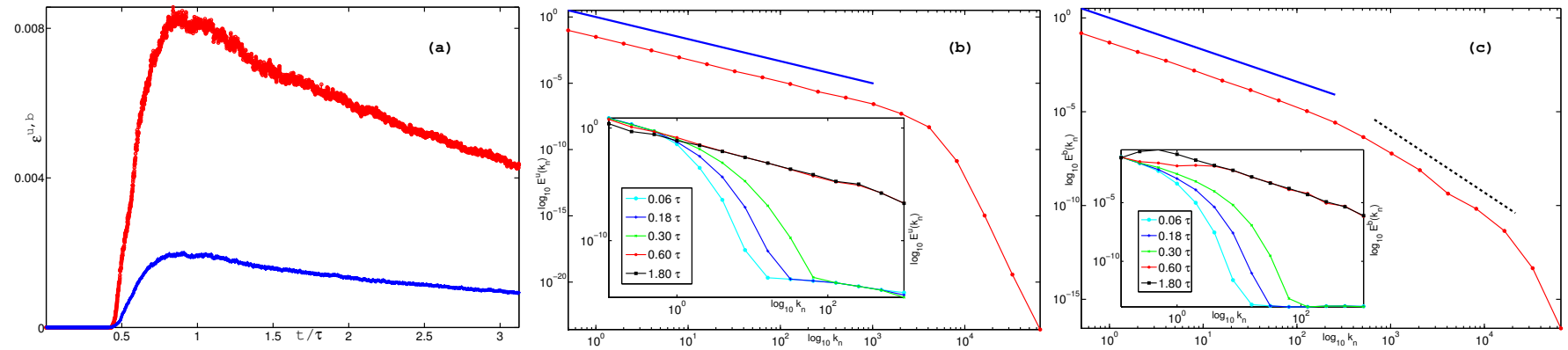

FIG. 5: (Color online) (a) Plots of $\varepsilon^{u}$ (red, upper curve) and $\varepsilon^{b}$ (blue, lower curve) versus the rescaled time $t / \tau$. (b) Plot of the kinetic-energy spectrum $E^{u}(k)$; the thick, blue line indicates the K41-scaling power law. Inset : Time evolution of $E^{u}(k)$ during the initial period with transients; the legend indicates the time at which each curve is obtained. (c) Plot of the magnetic-energy spectrum $E^{b}(k)$; the thick, blue line indicates K41 scaling in the low- $k$ region and the dashed, black line indicates $k^{-3.5}$ scaling in the high- $k$ region. Inset : Time evolution of $E^{b}(k)$ during the initial period with transients; the legend indicates the time at which each curve is obtained. The data shown are for run R3. 

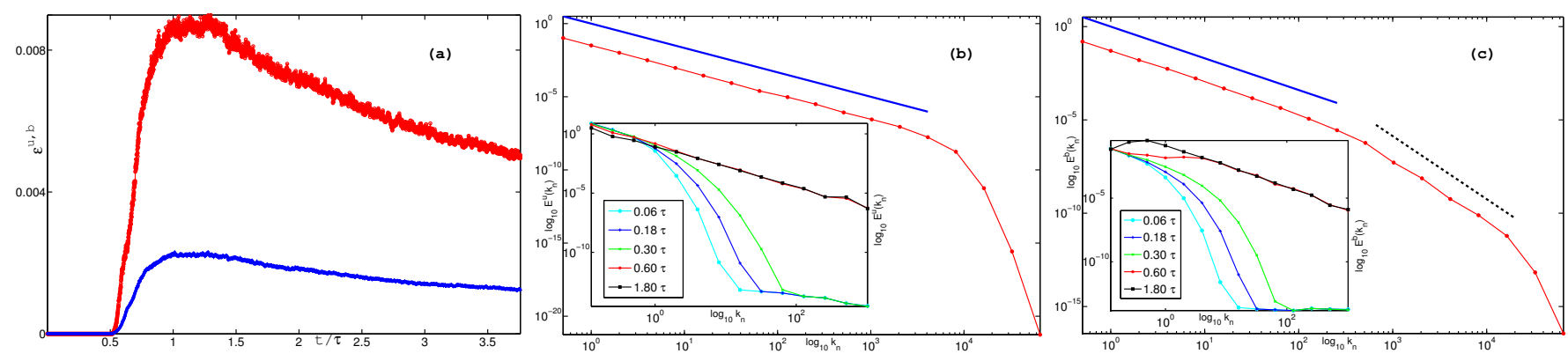

FIG. 6: (Color online) (a) Plots of $\varepsilon^{u}$ (red, upper curve) and $\varepsilon^{b}$ (blue, lower curve) versus the rescaled time $t / \tau$. (b) Plot of the kinetic-energy spectrum $E^{u}(k)$; the thick, blue line indicates the K41-scaling power law. Inset : Time evolution of $E^{u}(k)$ during the initial period with transients; the legend indicates the time at which each curve is obtained. (c) Plot of the magnetic-energy spectrum $E^{b}(k)$; the thick, blue line indicates K41 scaling in the low- $k$ region and the dashed, black line indicates $k^{-3.5}$ scaling in the high- $k$ region. Inset : Time evolution of $E^{b}(k)$ during the initial period with transients; the legend indicates the time at which each curve is obtained. The data shown are for run R4. 